\title{
Dynamics of vortices in weakly interacting Bose-Einstein condensates
}

\author{
Alexander Klein and Dieter Jaksch \\ Clarendon Laboratory, University of Oxford, Parks Road, Oxford OX1 3PU, United Kingdom \\ and Keble College, Parks Road, Oxford OX1 3PG, United Kingdom \\ Yanzhi Zhang and Weizhu Bao \\ Department of Mathematics and Center for Computational Science and Engineering, National University of Singapore, \\ Singapore 117543
}

(Received 3 August 2007; published 2 October 2007)

\begin{abstract}
We study the dynamics of vortices in ideal and weakly interacting Bose-Einstein condensates using a Ritz minimization method to solve the two-dimensional Gross-Pitaevskii equation. For different initial vortex configurations we calculate the trajectories of the vortices. We find conditions under which a vortex-antivortex pair annihilates and is created again. For the case of three vortices we show that at certain times two additional vortices may be created, which move through the condensate and annihilate each other again. For a noninteracting condensate this process is periodic, whereas for small interactions the essential features persist, but the periodicity is lost. The results are compared to exact numerical solutions of the Gross-Pitaevskii equation confirming our analytical findings.
\end{abstract}

DOI: 10.1103/PhysRevA.76.043602

PACS number(s): 03.75.Kk, 03.75.Lm, 05.30.Jp, 32.80.Pj

\section{INTRODUCTION}

Quantized vortices play an important role in verifying the superfluid properties of quantum liquids such as BoseEinstein condensates (BECs) or degenerate Fermi gases. In weakly interacting alkali gases condensate states containing a single vortex line were first created using Raman transition phase-imprinting methods [1]. By rotating the system with a laser spoon [2,3], vortex lattices containing more than 100 vortices have been created [4,5], and by using topological phase engineering methods [6] it is even possible to create multiply charged vortices. It is expected that more complicated vortex clusters can be created in the future, e.g., with the further development of phase-imprinting methods. Such states would enable various opportunities, ranging from investigating the properties of random polynomials [7] to using vortices in quantum memories [8]. All of these developments stir a great interest in the study of states with several vortices.

Recently, there were a number of investigations on the properties of vortices in BECs. For three-dimensional condensates, several studies on the dynamics of vortex lines have been done $[9,10]$. The generation and dynamics of vortices in a toroidal condensate have been investigated in Ref. [11], whereas detailed numerical studies of the optical generation of vortices in pancake-shaped condensates have been carried out in [12]. The manipulation of vortices such as charge conversion by external potentials has been discussed in Ref. [13]. Further numerical studies revealed that for condensates with a strong nonlinearity there exist several configurations of vortices which are stable [14-16]. Analytical expressions for the angular momentum and the energy of a vortex-antivortex configuration in a BEC have been obtained [17] using the Thomas-Fermi approximation. For strongly nonlinear condensates analytical solutions were derived by splitting the wave function into a region close to the vortex core and one far away from the vortex, where the hydrodynamic properties of the condensate are an essential feature
[18-20]. In contrast, here we concentrate on the dynamical properties of vortex configurations in weakly interacting condensates, and solutions for the whole spatial regime are obtained. In a noninteracting condensate the vortices behave similar to those created in an optical beam using holograms $[21,22]$, where the time evolution in the BEC corresponds to the spatial evolution of the laser beam. As we will show, the interaction between the BEC atoms changes the behavior of the vortex dynamics considerably.

In this paper, we make use of the Gross-Pitaevskii equation (GPE), also known as the nonlinear Schrödinger equation, which is known to be a valid description of the meanfield dynamics of a BEC at zero temperature. We consider a harmonic trap with tight confinement along one direction, such that the condensate is effectively two dimensional. For the case of an ideal, i.e., noninteracting BEC, the dynamics of the vortices is solved analytically yielding the essential features of the time evolution. For a small interaction within the condensate, we make use of the Ritz method in order to get analytical estimates of the dynamics. These estimates are compared to exact numerical solutions of the GPE using the time-splitting spectral method (TSSP), which is explicit, unconditionally stable and spectrally accurate in space. Details of the numerical method are described in Refs. [23-25].

This paper is organized as follows. In Sec. II we introduce the model under investigation and define the general initial states of the vortex configurations. In Sec. III we discuss the dynamics of vortices in an ideal condensate as a background for the results of Sec. IV, where a detailed investigation of the dynamics of a single vortex, a vortex pair, a vortex dipole, and a vortex tripole are presented. We conclude in Sec. V. An Appendix contains some more details on the evolution of a vortex tripole for the noninteracting BEC.

\section{THE MODEL}

In this work, we consider a Bose-Einstein condensate $(\mathrm{BEC})$ in a radially symmetric trap $V_{t}(x, y, z)=\frac{1}{2} m_{b}\left[\omega\left(x^{2}\right.\right.$ 
$\left.\left.+y^{2}\right)+\omega_{z} z^{2}\right]$ with $\omega_{z} \gg \omega$ the trap frequencies in axial and radial direction, respectively, and $m_{b}$ the mass of the BEC atoms. We assume a tight confinement in axial direction such that $\hbar \omega_{z} \gg k_{B} T$, where $k_{B}$ is Boltzmann's constant and $T$ is the temperature of the BEC, as well as $\hbar \omega_{z} \gg g n_{0}$, with $n_{0}$ the density of the BEC in the center of the trap and $g$ the interaction strength within the BEC, given by $g$ $=4 \pi \hbar^{2} a_{s} / m_{b}$, with $a_{s}$ the $s$-wave scattering length. For temperatures well below the critical temperature of the BEC and the Berezinskii-Kosterlitz-Thouless transition temperature $[26,27]$, a regime which is in reach of current experiments $[28,29]$, phase fluctuations occur on scales which are typically larger than the size of the condensate and the dynamics of the BEC is well described by the dimensionless 2D GrossPitaevskii equation [23]

$$
i \frac{\partial \psi}{\partial t}=\left[-\frac{1}{2}\left(\frac{\partial^{2}}{\partial x^{2}}+\frac{\partial^{2}}{\partial y^{2}}\right)+\frac{1}{2}\left(x^{2}+y^{2}\right)+\beta|\psi|^{2}\right] \psi .
$$

Here, $\psi=\psi(x, y, t)$ is the normalized wave function of the condensate with $\int|\psi(x, y)|^{2} d x d y=1$, and $\beta$ $=2 N a_{s} \sqrt{2 \pi \omega_{z} / \omega} / a_{0}$ characterizes the interatomic interaction, defined in terms of the total number of particles $N$ in the condensate. The above dimensionless quantities are obtained by scaling the length by the harmonic oscillator length $a_{0}$ $=\sqrt{\hbar / m_{b} \omega}$, the time by $\omega^{-1}$, and the energy by $\hbar \omega$.

We study the dynamics of $n$ vortices with topological charge $q_{j}= \pm 1 \quad(j=1,2, \ldots, n)$, which are initially placed at positions $\mathbf{r}_{j}=\left(x_{j}, y_{j}\right)$. For this purpose, we first need to calculate the ground state $\psi_{\mathrm{gs}}(\mathbf{r})$ of the GPE and the state $\psi_{q}(\mathbf{r})$ with a single vortex in the center of the trap. From this, we extract the function $p_{q}(\mathbf{r})=\psi_{q}(\mathbf{r}) / \psi_{\mathrm{gs}}(\mathbf{r})$, which describes a vortex in the BEC background. The initial state for the vortices is then approximately given by

$$
\psi(\mathbf{r}, t=0)=\alpha \psi_{\mathrm{gs}}(\mathbf{r}) \prod_{j=1}^{n} p_{q_{j}}\left(\mathbf{r}-\mathbf{r}_{j}\right),
$$

where $\alpha$ is chosen such that the initial state is normalized to 1. Unless otherwise stated, this normalization constant will be dropped in the following. The above approximation holds for vortices which are not too close to the edge of the condensate. A physical realization of such states can be achieved either by stirring the condensate $[2,3]$ or, in a more controlled way, by phase imprinting methods $[1,6]$ or by using light with orbital angular momentum [8].

\section{VORTICES IN A NONINTERACTING BEC}

To get an insight into the dynamics of vortices and as a background against the results found for the weakly interacting condensate we first focus on the noninteracting case $\beta$ $=0$, where the GPE simplifies to a two-dimensional harmonic oscillator. The initial state $\psi(\mathbf{r}, t=0)$ is expanded in terms of the solutions

$$
\psi_{n, m}(\mathbf{r}, t)=\frac{e^{-i E_{n, m^{t}}-\left(x^{2}+y^{2}\right) / 2}}{\sqrt{2^{n+m} n ! m ! \pi}} H_{n}(x) H_{m}(y)
$$

of the harmonic oscillator, with the energies $E_{n, m}=1+n+m$, $H_{n}$ are Hermite polynomials, and integer numbers $n, m \geq 0$.
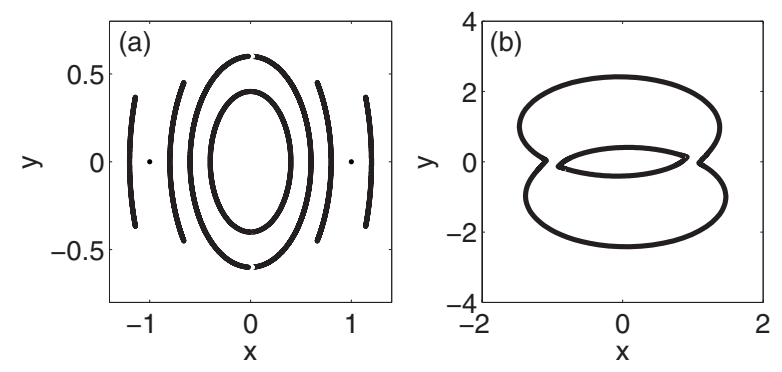

FIG. 1. (a) Trajectories for a vortex dipole for different initial positions $\left( \pm x_{0}, 0\right)$. From inside to outside $x_{0}=0.4,0.6,0.8,1,1.2$. For $x_{0}=0.4,0.6$, the two vortices annihilate each other at certain times and reappear again, indicated by the closed lines. For all other cases shown, there are always two vortices present apart from times where $t=(2 n+1) \pi / 2$, with $n$ an integer number. (b) Trajectories for the more general initial condition $\mathbf{r}_{1}=(0.9,0.1), \mathbf{r}_{2}=(-1.1,0)$.

This also gives the time evolution of the vortex state. The trajectories of the vortices are calculated by finding the zeros of the wave function and checking if at these points the condensate has a nonzero winding number.

For a single vortex of topological charge $q=+1$ initially located at $\left(x_{1}, 0\right)$ the expansion gives the wave function

$$
\phi_{v}(x, y, t)=\exp \left(-\frac{x^{2}+y^{2}}{2}-2 i t\right)\left(x+i y-e^{i t} x_{1}\right) .
$$

The position of the vortex evolves in time on an exact circular trajectory described by $x_{v}(t)=x_{1} \cos (t)$ and $y_{v}(t)$ $=x_{1} \sin (t)$.

For a vortex pair, that is two vortices with identical topological charge $q=+1$ initially located at $\left(x_{j}, y_{j}\right)$, where $j$ $=1,2$, the expansion into the solutions of the harmonic oscillator shows that the two vortices move independently from each other on trajectories $x_{v p, j}(t)=x_{j} \cos (t)-y_{j} \sin (t)$ and $y_{v p, j}(t)=x_{j} \sin (t)+y_{j} \cos (t)$, which are exactly the same as for a single vortex. Due to the conservation of the total topological charge $Q=\sum_{j} q_{j}$ there are always at least two vortices present in the condensate, since a doubly charged vortex is unstable [30]. From the absence of additional vortices we conclude that the total energy of the system is not large enough to allow the spontaneous creation of a vortexantivortex pair.

The dynamics changes considerably for a vortex dipole. Assuming the $q=+1$ vortex is initially located at $\left(x_{0}, 0\right)$ and the $q=-1$ vortex at $\left(-x_{0}, 0\right)$, the trajectories are given by $y_{v d}(t)=\sin (t)\left(x_{0}^{2}-1\right) / x_{0}$ and $x_{v d}(t)= \pm \sqrt{x_{0}^{2}-y_{v d}^{2}(t)}$. Examples of these trajectories are shown in Fig. 1(a). For $x_{0} \geq 1 / \sqrt{2}$ these expressions are always real numbers indicating that the two vortices do not annihilate each other. For $x_{0}=1$ the vortices remain even stationary at their initial positions. The situation changes for $x_{0}<1 / \sqrt{2}$. In this case, the vortices collide with each other at a time $t_{a}$ given by the first possible solution of

$$
|\sin (t)|=\left|\frac{x_{0}^{2}}{x_{0}^{2}-1}\right| .
$$

They annihilate each other and only reappear again at time $t_{r}$, where the second possible solution of Eq. (5) in the half- 
period $t \in[0, \pi]$ is taken. Between those two times, there are no vortices present in the condensate. We also did calculations with more general initial conditions for the two vortices, located at $\mathbf{r}_{j}=\left(x_{j}, y_{j}\right)$, as shown in Fig. 1(b). The whole dynamics is still periodic with a period of $2 \pi$, and after a time of $t=\pi$ the state is given by $\phi_{v d}(x, y, \pi)=-\phi_{v d}(-x$, $-y, 0)$, which means the initial state is, up to an unimportant global phase, inflected at the origin. For the symmetric stable case with $x_{0}=1$ this might be surprising at the first glance, but is explained by the fact that the (dimensionless) current density $\mathbf{j}(x, y, t)=-i\left(\psi^{*} \nabla \psi-\psi \nabla \psi^{*}\right) / 2$ stops at times $t=(2 n$ $+1) \pi / 2, n=1,2, \ldots$, allowing the two stationary vortices to flip their signs.

The dynamics of a vortex tripole with two vortices of topological charge $q=+1$ at locations $\left(x_{0}, 0\right)$ and $\left(-x_{0}, 0\right)$ and one of charge $q=-1$ at $(0,0)$ is given by

$$
\begin{aligned}
\phi_{v t}(x, y, t)= & e^{-\left[\left(x^{2}+y^{2}\right) / 2\right]-4 i t}\left\{x^{3}+i y x^{2}+\left[y^{2}-e^{2 i t}\left(x_{0}^{2}-2\right)-2\right] x\right. \\
& \left.+i y\left[y^{2}+e^{2 i t}\left(x_{0}^{2}+2\right)-2\right]\right\} .
\end{aligned}
$$

For the special case $x_{0}=\sqrt{2}$, we find as the zeros of the wave function $(0,0), \quad(-\sqrt{2}, 0), \quad(\sqrt{2}, 0), \quad(\sqrt{2-4 \cos (2 t)} \sin (2 t)$, $-\sqrt{2-4 \cos (2 t)} \cos (2 t)), \quad$ and $\quad(-\sqrt{2-4 \cos (2 t)} \sin (2 t)$, $\sqrt{2-4 \cos (2 t)} \cos (2 t))$. Although the initial state only contains three vortices, during the evolution additional vortices are created and annihilated again, so that at certain times there is a maximum of five vortices present in the condensate. The whole evolution is periodic with a period of $\pi$, a property which will be lost for small interactions. For more details of the vortex dynamics in the ideal case we refer to the Appendix.

\section{VORTICES IN A WEAKLY INTERACTING BEC}

The situation gets more complicated when a finite interaction $\beta$ of the condensate is taken into account, and exact analytical solutions are not known. In order to calculate the dynamics of the vortices we therefore proceed using the Ritz minimization method [31]. For small interactions $\beta \leq 1$ we assume that the Gaussian shape of the condensate is not changed, but only broadened. For the solutions of the GrossPitaevskii equation we make the ansatz

$$
\psi_{n, m, \beta}(\mathbf{r}, t)=\frac{e^{-i \mu_{n, m^{t}}-\left[\left(x^{2}+y^{2}\right) / 2 \sigma^{2}\right]}}{\sqrt{2^{n+m} n ! m ! \pi \sigma^{2}}} H_{n}(x / \sigma) H_{m}(y / \sigma),
$$

where the functions are normalized to 1 and $\sigma$ is a constant which takes the broadening into account. This constant is derived by minimizing the Gross-Pitaevskii energy functional [30]

$$
E[\psi]=N \int d \mathbf{r} \frac{1}{2}|\nabla \psi|^{2}+\frac{1}{2} \mathbf{r}^{2}|\psi|^{2}+\frac{\beta}{2}|\psi|^{4}
$$

with respect to $\sigma$, where $\psi=\psi_{0,0, \beta}(\mathbf{r}, 0)$ and the energy is given in units of $\hbar \omega$. The minimum is found for $\sigma^{2}$ $=\sqrt{(\beta+2 \pi) / 2 \pi}$. The constants $\mu_{n, m}$ are derived by putting the ansatz into the GPE (1), multiplying by $\psi^{*}$ and integrating over space, i.e.,
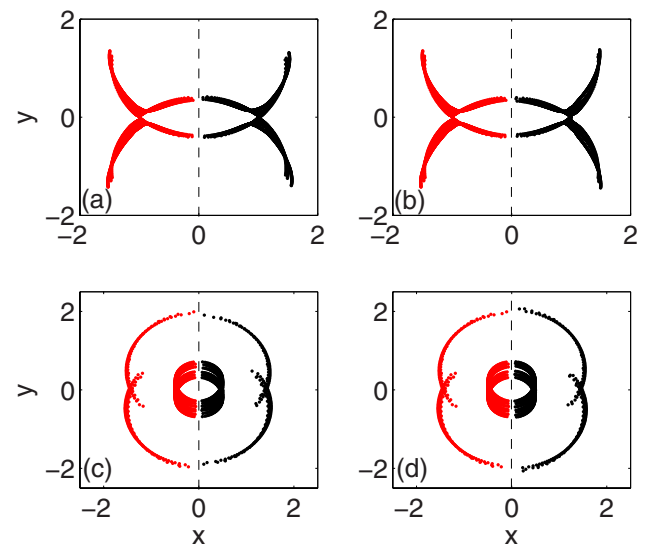

FIG. 2. (Color online) Comparison of vortex trajectories for different initial states for a vortex dipole with initial $x_{0}=1$ [(a) and (b)] and $x_{0}=0.5[$ (c) and (d)]. For $x<0$ the trajectories for a vortex dipole with initial $p_{q}$ for $\beta=1$ is shown, whereas for $x>0$ the trajectories with initial $p_{q}$ for $\beta=0[(\mathrm{a})$ and (c)] and $\beta=2[(\mathrm{~b})$ and (d)] are plotted. All trajectories are mirror symmetric to the axis where $x=0$ (dashed line). The vortices were evolved for a time interval $[0,20]$ and a condensate with $\beta=1$.

$$
\mu_{n, m}=\int d \mathbf{r} \frac{1}{2}\left|\nabla \psi_{n, m, \beta}\right|^{2}+\frac{1}{2} \mathbf{r}^{2}\left|\psi_{n, m, \beta}\right|^{2}+\beta\left|\psi_{n, m, \beta}\right|^{4} .
$$

The wave functions $\psi_{n, m, \beta}(\mathbf{r}, t)$ are then used to expand the vortex state, which is given by the functions describing the vortices multiplied by $\psi_{0,0, \beta}$ and a normalization factor. This factor is, as in the preceding sections, dropped unless otherwise stated.

We have further assumed that the shape of the vortices does not change due to the increased interaction. Normally, in a strongly nonlinear condensate the size of a vortex is given by the coherence length $\xi$, for which we find (in scaled units) $\xi^{2}=\sqrt{\pi / 4 \beta}$. However, for our trapped weakly interacting condensate this length scale is no longer useful, which gets especially apparent for a vanishing interaction $\beta \rightarrow 0$, leading to $\xi \rightarrow \infty$. Instead by using numerical calculations we find that the vortices are still well described by the functions $p_{q}$ derived for $\beta=0$. This is illustrated in Figs. 2(a) and 2(c), where the trajectories for a vortex dipole with the analytical $p_{q}$ for $\beta=0$ are compared to those with numerically found $p_{q}$ for $\beta=1$. The difference between the trajectories is negligible. In Figs. 2(b) and 2(d) we compare the vortex trajectories for the factor $p_{q}$ found for $\beta=1$ and $\beta=2$. Again, the trajectories are almost identical. This shows that a small perturbation in the shape of the vortices leaves the trajectories essentially unaffected as long as an initial condition described by Eq. (2) is used. We note that Eq. (2) only describes initially factorized vortices, which are not necessarily preferred as the initial states with a given number of vortices. Alternative states can be considered, and it has been shown that the dynamics for globally linked, that means nonfactorized, vortices can behave significantly different from the dynamics of factorized ones [32]. However, a full characterization of such states is beyond the scope of this paper and we therefore restrict our considerations to initial states described by Eq. (2). 

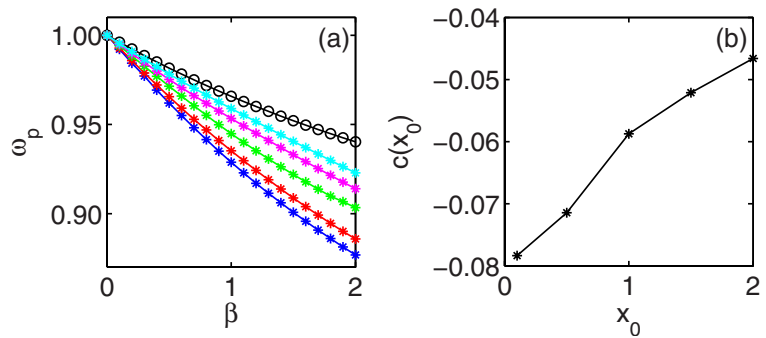

FIG. 3. (Color online) (a) Precession frequency of a single vortex versus the interaction strength $\beta$. Stars show $\omega_{p}$ for a different initial distance of the vortex from the center, namely (from bottom to top) $x_{0}=0.1,0.5,1.0,1.5,2.0$. The circles show the analytical result given in Eq. (13), lines are guides to the eye. (b) The coefficients $c\left(x_{0}\right)$ for different $x_{0}$, see text.

\section{A. Single vortex}

For a single vortex initially located at $\left(x_{0}, 0\right)$ the expansion into the solutions $\psi_{n, m, \beta}$ yields

$$
\begin{aligned}
\phi_{\mathrm{v}}(x, y, t)= & e^{-\{[i(7 \beta+16 \pi) t] / 4 \sqrt{2 \pi} \sqrt{\beta+2 \pi}\}-\left[\left(x^{2}+y^{2}\right) / 2 \sigma^{2}\right]} \\
& \times\left(x-e^{[i(\beta+8 \pi) t] / 4 \sqrt{2 \pi} \sqrt{\beta+2 \pi}} x_{0}+i y\right),
\end{aligned}
$$

which immediately leads to the trajectory

$$
\begin{aligned}
& x_{v}(t)=x_{0} \cos \left(\frac{(\beta+8 \pi) t}{4 \sqrt{2 \pi} \sqrt{\beta+2 \pi}}\right), \\
& y_{v}(t)=x_{0} \sin \left(\frac{(\beta+8 \pi) t}{4 \sqrt{2 \pi} \sqrt{\beta+2 \pi}}\right) .
\end{aligned}
$$

The vortex again moves on an exact circular line, however the time it needs to complete one circle is increased compared to the interaction-free case. To be more specific, the precession frequency is given by

$$
\omega_{p}=\frac{(\beta+8 \pi)}{4 \sqrt{2 \pi} \sqrt{\beta+2 \pi}} .
$$

A comparison with numerical results shown in Fig. 3(a) illustrates that the analytical result describes the trend of a decreasing precession frequency well, however, the numerical results show a clear dependence on the distance $x_{0}$ of the vortex to the center of the trap, which is missing in the analytical formula. For small interaction $\beta$ the behavior of the precession frequency can be assumed to be linear with $\beta$ and follow the curve $\omega_{p}=1+c\left(x_{0}\right) \beta$. For the analytical formula we get $c_{\text {ana }}\left(x_{0}\right)=-1 / 8 \pi \approx-0.04$, whereas the results from the numerics are shown in Fig. 3(b). A trend towards the analytical value is visible with increasing $x_{0}$. However, measuring the frequency for $x_{0}>2$ will become increasingly difficult due to the dilute condensate density for large distances.

\section{B. Vortex pair}

Let us assume that at time $t=0$ the two vortices with topological charge $q_{j}=+1$ are located at $\left(-x_{0}, 0\right)$ and $\left(x_{0}, 0\right)$. The time evolution of the BEC wave function is given by

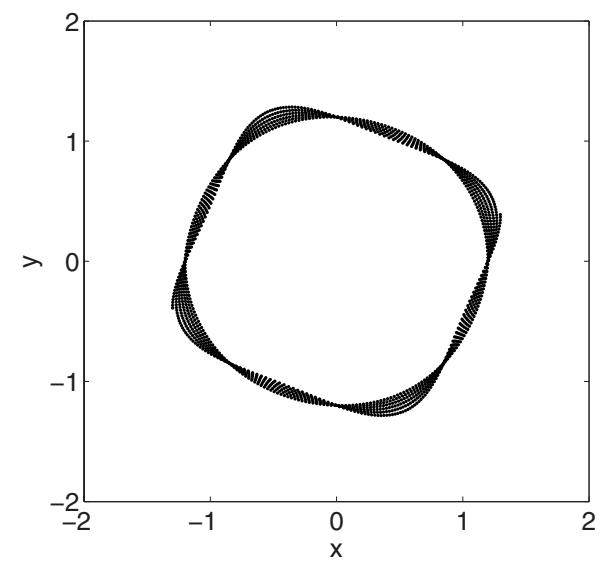

FIG. 4. Trajectories of a vortex pair deduced from the analytical formula Eq. (14) for $\beta=2, x_{0}=1.2$, and a time interval of $t$ $\in[0,20]$. The trajectories are no longer exact circular lines, indicating that both vortices influence each other.

$$
\begin{aligned}
\phi_{v p}= & e^{-\{[i(137 \beta+384 \pi) t] / 64 \sqrt{2 \pi} \sqrt{\beta+2 \pi}\}-\left[\left(x^{2}+y^{2}\right) / 2 \sigma^{2}\right]} \\
& \times\left(x^{2}+2 i e^{(5 i \beta t) / 64 \sqrt{2 \pi} \sqrt{\beta+2 \pi}} y x\right. \\
& \left.-e^{[i(41 \beta+256 \pi) t] / 64 \sqrt{2 \pi} \sqrt{\beta+2 \pi}} x_{0}^{2}-y^{2}\right) .
\end{aligned}
$$

We see that the factor describing the two vortices in general cannot be factorized as in the interaction-free case, indicating that the two vortices influence each other. This also becomes evident when investigating the trajectories of the two vortices. They no longer move on exact circular lines, but rather on deformed ones as shown in Fig. 4. The deviation from the exact circular line gets larger for higher interaction strength $\beta$.

\section{Vortex dipole}

For symmetric initial conditions, i.e., a negative vortex initially at position $\left(-x_{0}, 0\right)$ and a positive one at position $\left(x_{0}, 0\right)$, the wave function for a vortex dipole is expanded in terms of $\psi_{n, m, \beta}$ yielding

$$
\begin{aligned}
\phi_{v d}(\mathbf{r}, t)= & e^{\{[-3 i(115 \beta+256 \pi) t] / 64 \sqrt{2 \pi} \sqrt{\beta+2 \pi}\}-\left[\left(x^{2}+y^{2}\right) / 2 \sigma^{2}\right]} \\
& \times\left(2 i e^{[i(233 \beta+512 \pi) t] / 64 \sqrt{2 \pi} \sqrt{\beta+2 \pi}} x_{0} y\right. \\
& +e^{[i(249 \beta+640 \pi) t] / 64 \sqrt{2 \pi} \sqrt{\beta+2 \pi}}\left(\sigma-x_{0}\right)\left(\sigma+x_{0}\right) \\
& \left.+e^{[i(13 \beta+24 \pi) t] / 4 \sqrt{2 \pi} \sqrt{\beta+2 \pi}}\left(-\sigma^{2}+x^{2}+y^{2}\right)\right) .
\end{aligned}
$$

As for the vortex pair the dynamics of the BEC is no longer periodic due to the interaction, but acquires a more complicated time dependence. As an example, the trajectories for $\beta=1$, a time interval of $t \in[0,20]$, and several initial positions $\left( \pm x_{0}, 0\right)$ are shown in Fig. 5 . The simple trajectories from the noninteracting case are changed to complicated structures, which have lost their periodicity.

Comparison between analytical results using Eq. (15) and numerics shows that especially for small times and intermediate distances $2 x_{0} \approx 2$ both trajectories agree quite well, however for certain times the analytical results predict vortices at positions where there should be no vortices according 

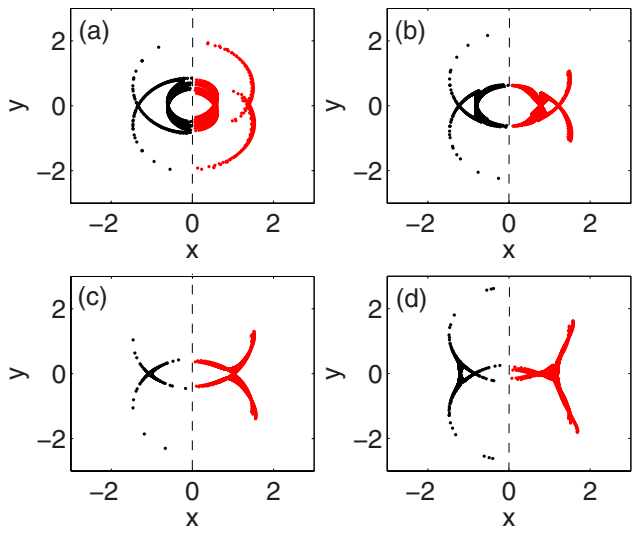

FIG. 5. (Color online) Trajectories of the two vortices in a vortex dipole for (a) $x_{0}=0.6$, (b) $x_{0}=0.8$, (c) $x_{0}=1$, and (d) $x_{0}=1.2$. Analytical results calculated with Eq. (15) are only shown for $x$ $<0$, whereas numerical results are only shown for $x>0$. Both results are mirror symmetric with respect to the axis where $x=0$ (dashed line). The interaction is chosen as $\beta=1$, and the time interval for which the trajectories are shown is given by $0 \leq t \leq 20$.

to numerics. The differences between analytics and numerics get larger for increasing interaction $\beta$. Our numerical calculations furthermore suggest that even for distances $2 x_{0}>2$ there exist times for which the two vortices annihilate each other and reappear again, so, for example, at $2 x_{0}=3$ and a time around $t=18$. This is in contrast to the noninteracting case, where the evolution was strictly periodic and an annihilation of the vortices not possible if their initial distance was $2 x_{0}>2 / \sqrt{2}$.

\section{Vortex tripole}

For a vortex tripole as introduced in Sec. III the wave function describing the time evolution is given by

$$
\begin{aligned}
\phi_{v t}(x, y, t)= & e^{-[(871 i \beta t) / 128 \sqrt{2 \pi} \sqrt{\beta+2 \pi}]-\left\{\left[\left(20 i \sqrt{2 \pi} t \sigma^{2} / \sqrt{\beta+2 \pi}\right)+x^{2}+y^{2}\right] / 2 \sigma^{2}\right\}} \\
& \times\left(e ^ { [ 3 i ( 3 6 9 \beta + 1 0 2 4 \pi ) t ] / 2 5 6 \sqrt { 2 \pi } \sqrt { \beta + 2 \pi } } \left[\sigma^{2}(x+i y)\right.\right. \\
& -2 i x(x-i y) y]+e^{[3 i(361 \beta+1024 \pi) t] / 256 \sqrt{2 \pi} \sqrt{\beta+2 \pi}} \\
& \times\left[3 \sigma^{2}(x+i y)-2\left(x^{3}+i y^{3}\right)\right] \\
& -2 e^{[i(647 \beta+2048 \pi) t] / 128 \sqrt{2 \pi} \sqrt{\beta+2 \pi}}\left[2 \sigma^{2}(x+i y)\right. \\
& \left.\left.-(x-i y) x_{0}^{2}\right]\right) .
\end{aligned}
$$

As for the noninteracting case, there always exists a vortex in the center of the condensate, however we were not able to identify any initial condition where more than the central vortex are stationary. This is consistent with the results reported in Ref. [16]. There it was shown that a stable vortex tripole, i.e., a configuration of exactly three stationary vortices, only exists for interactions $\beta \gg 1$, where our ansatz is no longer valid.

The zeros of the wave function Eq. (16) are found numerically for different initial positions of the vortices. For short times $t \leq 10$, the time evolution of the trajectories is quasiperiodic, however this quasiperiodicity is more and more washed out for longer times. This gets also apparent
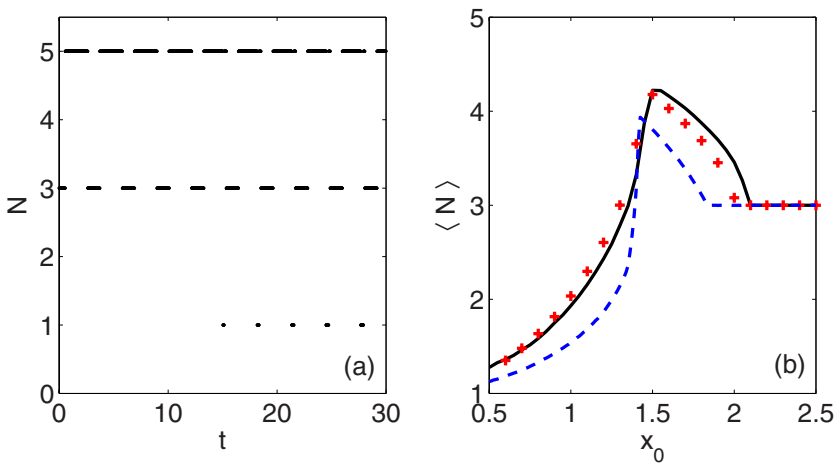

FIG. 6. (Color online) (a) Number of vortices $N$ present in the BEC vs time, with initially $x_{0}=1.5$. (b) Average number of vortices $\langle N\rangle$ present in the BEC during the time interval $[0,20]$ for different initial $x_{0}$. The solid line shows the results derived from the analytical expression, Eq. (16), whereas the pluses show numerical results. In all cases we have chosen $\beta=1$, apart from the dashed line, which shows the results for $\beta=0$.

when the number of vortices during time is considered. As shown in Fig. 6(a), the number of vortices oscillates for $t$ $\leq 10$ between 3 and 5 for an initial configuration with $x_{0}$ $=1.5$. For later times, however, there are intervals in which the condensate only exhibits one vortex.

In Fig. 6(b) we show the average number of vortices $\langle N\rangle$ in the condensate during the time interval $[0,20]$ versus the initial positions $x_{0}$. The results derived from the numerical evolution of the GPE agree quite well with the ones from the analytical formula Eq. (16). We observe that for $x_{0} \lesssim 1.5$ the average number of vortices $\langle N\rangle$ increases with increasing $x_{0}$, and reaches a pronounced maximum at $x_{0} \approx 1$.5. For larger $x_{0}$ the average vortex number decreases again to reach a constant value of 3 for $x_{0} \geq 2$. This indicates that for such large distances the energy within the condensate is too low to spontaneously create additional vortices. This behavior is qualitatively similar to the noninteracting case as also indicated in Fig. 6(b), where the interacting case tends to exhibit a higher average vortex number.

\section{CONCLUSION}

In the present paper we have investigated the dynamics of vortices in two-dimensional Bose-Einstein condensates. We have solved the Gross-Pitaevskii equation analytically for ideal condensates and have used the Ritz minimization method in order to calculate the dynamics for small interactions. The latter results were compared to exact numerical solutions of the condensate dynamics.

For an ideal condensate we have shown that two vortices with the same topological charge $|q|=1$ do not influence each other and behave like two independent, single vortices. This changes as soon as the interaction within the condensate is taken into account. The two vortices no longer move on exact circular lines, but rather on a distorted circular path.

For the case of a vortex dipole, that is two vortices with opposite topological charge in the condensate, we found that for certain initial conditions in an ideal condensate the two 
vortices will collide, thereby annihilating each other, and reappear again, whereas they are always separated if the initial distance is large enough. The trajectories change considerably in an interacting condensate, and a large initial distance will no longer guarantee that the two vortices do not annihilate each other at some times.

We also investigated the case where initially there are three vortices in the BEC, a so-called vortex tripole. In contrast to the case of only two vortices present at the beginning we found that for an ideal condensate during the time evolution additional vortices were created and annihilated, allowing for a maximum of five vortices in the condensate. Our numerical results showed that this behavior also persisted in an interacting condensate.

\section{ACKNOWLEDGMENTS}

This work was supported by the National University of Singapore Grant No. R-146-000-083-112, the EPSRC (UK) through the QIP IRC (GR/S82176/01) and the EuroQUAM project EP/E041612/1, and by the EU through the STREP project OLAQUI. One of the authors (A.K.) acknowledges financial support from the Keble Association.

\section{APPENDIX: DETAILS OF THE VORTEX TRIPOLE DYNAMICS FOR THE IDEAL BEC}

The expressions given for the vortex trajectories in Sec. III do not include information on the topological charge of the respective vortices. Closer investigations show that the vortices described by a single trajectory can indeed flip their signs. To be more precise, we assume that at $t=0$ the central vortex has a negative charge. At times $t<\pi / 6$, there are only three vortices present in the BEC, which do not move. For $t=\pi / 6$, two new vortices of equal charge $q=-1$ arise in the center of the BEC flipping the charge of the central vortex in order to keep the total topological charge $Q$ constant. The two new vortices start to move out of the center towards the two stationary vortices. When the two new vortices cross the two stationary ones at $t=\pi / 4$, their charges are flipped as well, such that the moving vortices have now a positive charge and the stationary ones a negative one. After the flip,
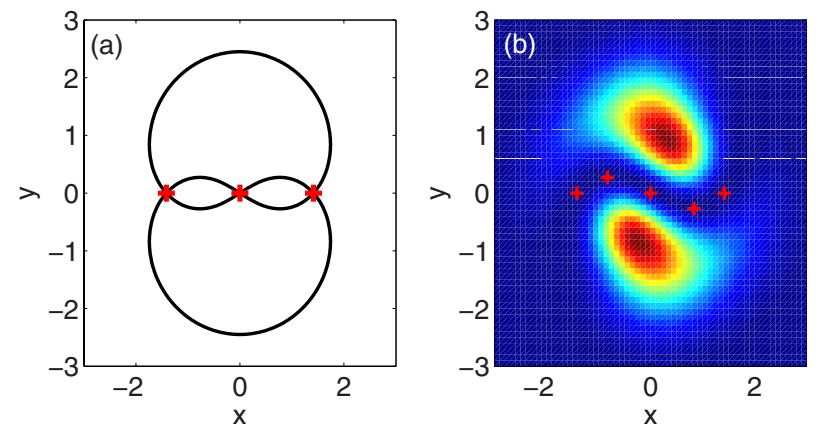

FIG. 7. (Color online) (a) Trajectories of the two additional vortices (black lines) for the initial state of a vortex tripole with $x_{0}=\sqrt{2}$, details see text. The three stationary vortices are indicated by plus signs. (b) Density plot for $t=0.2 \pi$. Plus signs indicate the position of the five vortices.

the nonstationary vortices move around the three stationary vortices as indicated in Fig. 7(a), cross the two vortices outside the center again and flipping their charge at $t=3 \pi / 4$, and finally annihilate at the center at a time $t=5 \pi / 6$, such that the original configuration for $t=0$ is achieved again. This is repeated periodically, with a period of $\pi$. Investigating the phase of the condensate at the times $t=\pi / 4$ and $3 \pi / 4$, where the moving vortices cross the stationary ones at $( \pm \sqrt{2}, 0)$, shows that for these times there is only one vortex present in the condensate, namely the central one. A density plot of the system for a time $t=0.2 \pi$ is shown in Fig. 7(b). The additional vortices are created in a region of low density, where the energetic cost is lowest. However, this low density makes the measurement of the vortices more difficult.

For the case $x_{0} \neq \sqrt{2}$ we find a similar behavior of the vortices. For $x_{0} \geq 2$ there will always be three vortices in the condensate. For smaller values, additional vortices can be created, and for $x_{0}<\sqrt{2}$ we find that for certain time intervals (i.e., not only for single points in time) there is only one vortex present in the condensate. Due to the conservation of the total topological charge this is the minimum number of vortices present. Calculating the roots of Eq. (6) shows that the maximum number of vortices is five.
[1] M. R. Matthews, B. P. Anderson, P. C. Haljan, D. S. Hall, C. E. Wieman, and E. A. Cornell, Phys. Rev. Lett. 83, 2498 (1999).

[2] K. W. Madison, F. Chevy, W. Wohlleben, and J. Dalibard, Phys. Rev. Lett. 84, 806 (2000).

[3] K. W. Madison, F. Chevy, W. Wohlleben, and J. Dalibard, J. Mod. Opt. 47, 2715 (2000).

[4] J. R. Abo-Shaeer, C. Raman, J. M. Vogels, and W. Ketterle, Science 292, 476 (2001).

[5] C. Raman, J. R. Abo-Shaeer, J. M. Vogels, K. Xu, and W. Ketterle, Phys. Rev. Lett. 87, 210402 (2001).

[6] A. E. Leanhardt, A. Görlitz, A. P. Chikkatur, D. Kielpinski, Y. Shin, D. E. Pritchard, and W. Ketterle, Phys. Rev. Lett. 89, 190403 (2002).
[7] Y. Castin, Z. Hadzibabic, S. Stock, J. Dalibard, and S. Stringari, Phys. Rev. Lett. 96, 040405 (2006).

[8] K. T. Kapale and J. P. Dowling, Phys. Rev. Lett. 95, 173601 (2005).

[9] B. Jackson, J. F. McCann, and C. S. Adams, Phys. Rev. A 61, 013604 (1999).

[10] D. L. Feder, A. A. Svidzinsky, A. L. Fetter, and C. W. Clark, Phys. Rev. Lett. 86, 564 (2001).

[11] J.-P. Martikainen, K.-A. Suominen, L. Santos, T. Schulte, and A. Sanpera, Phys. Rev. A 64, 063602 (2001).

[12] G. Andrelczyk, M. Brewczyk, Ł. Dobrek, M. Gajda, and M. Lewenstein, Phys. Rev. A 64, 043601 (2001).

[13] V. M. Pérez-García, M. A. García-March, and A. Ferrando, 
Phys. Rev. A 75, 033618 (2007).

[14] L.-C. Crasovan, V. Vekslerchik, V. M. Pérez-García, J. P. Torres, D. Mihalache, and L. Torner, Phys. Rev. A 68, 063609 (2003).

[15] M. Möttönen, S. M. M. Virtanen, T. Isoshima, and M. M. Salomaa, Phys. Rev. A 71, 033626 (2005).

[16] V. Pietilä, M. Möttönen, T. Isoshima, J. A. M. Huhtamäki, and S. M. M. Virtanen, Phys. Rev. A 74, 023603 (2006).

[17] Q. Zhou and H. Zhai, Phys. Rev. A 70, 043619 (2004).

[18] B. Y. Rubinstein and L. M. Pismen, Physica D 78, 1 (1994).

[19] L. M. Pismen, Vortices in Nonlinear Fields: From Liquid Crystals to Superfluids, From Non-Equilibrium Patterns to Cosmic Strings, 1st ed. (Oxford University Press, New York, 1999).

[20] J. R. Anglin, Phys. Rev. A 65, 063611 (2002).

[21] G. Molina-Terriza, L. Torner, E. M. Wright, J. J. García-Ripoll, and V. M. Pérez-García, Opt. Lett. 26, 1601 (2001).

[22] F. S. Roux, Opt. Commun. 234, 63 (2004).

[23] W. Bao, D. Jaksch, and P. A. Markovich, J. Comput. Phys.
187, 318 (2003)

[24] W. Bao and Y. Zhang, Math. Models Meth. Appl. Sci. 15, 1863 (2005).

[25] W. Bao and D. Jaksch, SIAM (Soc. Ind. Appl. Math.) J. Numer. Anal. 41, 1406 (2003).

[26] V. L. Berezinskii, Sov. Phys. JETP 34, 610 (1972).

[27] J. M. Kosterlitz and D. J. Thouless, J. Phys. C 6, 1181 (1973).

[28] Z. Hadzibabic, P. Krüger, M. Cheneau, B. Battelier, and J. Dalibard, Nature (London) 441, 1118 (2006).

[29] P. Krüger, Z. Hadzibabic, and J. Dalibard, Phys. Rev. Lett. 99, 040402 (2007).

[30] L. Pitaevskii and S. Stringari, Bose-Einstein Condensation (Clarendon, Oxford, 2003).

[31] C. Cohen-Tannoudji, B. Diu, and F. Laloë, Quantum Mechanics (Wiley-Interscience, New York, 1977).

[32] L.-C. Crasovan, G. Molina-Terriza, J. P. Torres, L. Torner, V. M. Pérez-García, and D. Mihalache, Phys. Rev. E 66, 036612 (2002). 